\title{
How and how much to invest for fighting cheaters: from an ODE to a Cellular Automata model
}

\author{
Luca Meacci $^{1}$
}

Received: 8 October 2014/Accepted: 17 June 2015/Published online: 1 July 2015

(c) The Author(s) 2015. This article is published with open access at Springerlink.com

\begin{abstract}
In this paper, we present a study which is the completion of the problem left open in the appendix of the paper Nuño et al. (Eur J Appl Math 21:459-478, 2010). The goal of the paper is to offer a deeper analysis of the type of the ODE system featured in the paper above concerning the evolution of the total wealth and cheater population and, mainly, the purpose is to generalize this one to a Cellular Automata model introducing spatial and local effects. The work presented shows the original interaction between an Ordinary Differential Equations model and a Cellular Automata model.
\end{abstract}

Keywords Mathematical modeling - ODE equations . Cellular Automata model · Tax evasion

Mathematics Subject Classification $34 \mathrm{~A} 34 \cdot 37 \mathrm{~B} 15$. $68 \mathrm{Q} 80 \cdot 68 \mathrm{U} 20 \cdot 91 \mathrm{D} 10 \cdot 91 \mathrm{D} 25$

\section{Introduction}

An ideal society is composed by citizens whom all contribute to the common wealth. The common wealth can be used for education, public buildings and for all the needs of the population. But unfortunately in each real society there are always people that do not contribute to the common wealth but benefit from it, the cheaters. This is a form of criminal behavior that can create serious problems in each society (see $[1,2])$.

Luca Meacci

luca.meacci@gmail.com

1 Dipartimento di Matematica, "Ulisse Dini”, Università degli Studi di Firenze, Viale Morgagni, 67/A, 50134 Florence, Italy
How can a society organize itself to fight this social threat? In particular, how and how much can the common wealth be spent to fight cheaters?

In this paper, we try to answer these questions using simple models. Recent publications show the growing interest in the applications of mathematics in preventing tax evasion and crime [3,4]. The present work is organized essentially in two parts. In the first part, mathematical modeling provides a system of Ordinary Differential Equations, as in the appendix of work [5]. We studied the system by tuning the parameters and solved it. In the second part, we generalized the model in a 2D system using the method of cellular automaton, a sort of agentbased model. We confronted the results with the previous model, and then we studied the effects that arose from assuming different polities in different zones of the world.

Our decisions depend on the global factors that are related to the whole society. Examples are the social forces, defined as external factors which have bias opinions, that include political advertisements, news reports, laws and sanctions. These elements are called "macro-sociological" components [6], because their influence is a characteristic of the entire system. These factors are those which are involved in a homogeneous system, of which we take into account in an ODE system. On the other hand, each member of a population is influenced by the other people whom each one interacts with. For example, our behavior is influenced by the opinions and the actions of our neighbors and friends. These influences no longer belong to the whole system, but they are local elements involved in the so-called "micro-sociological" level [7]. For these reasons, we have to take in account the effects of the socalled "micro-sociology" and "macro-sociology", to completely describe the evolution of the society. What happens when we introduce these local influences? Our 
aim is to build a Cellular Automata model, consistent with the ODE model, which allows it to describe the spatial effects due to local influences at the "micro-sociological" level.

\section{Homogeneous case}

Let us assume that a society is composed by the following two classes:

1. $X:=$ people that contribute to common wealth (taxpayers),

2. $\quad Y:=$ people that do not contribute (cheaters).

We suppose that the total population is constant, i.e.,

$N=X+Y$.

Let us define the common wealth, $W$, like wealth sustained by the contributions of law-abiding citizens and from which all people benefit. This common wealth is also used to convince cheaters to contribute to $W$ with policies of repression and education. The time evolution of $W$ is given by

$\dot{W}=a(N-Y)-\theta W-\phi W$.

The common wealth increases in proportion to the number of taxpayers ( $a$ is the constant of proportion) and decreases in proportion with the expenditures. A part of the expenditures is due to fight cheaters. The parameter $\phi$ takes into account these kinds of measures while all other expenditures of the society are expressed by parameter $\theta$.

The time evolution of cheater population, according to the classical population dynamics [8], is

$\dot{Y}=\tau(N-Y)-\alpha Y$.

The rates $\tau$ and $\alpha$ are, respectively, influenced by social promotion and police repression (arrests). This can be expressed postulating

$\tau=\frac{\tau_{0}}{1+\tau_{1} s \phi W}$

and

$\alpha=\alpha_{0}+\alpha_{1}(1-s) \phi W$

where $\phi W$ is the fraction of the total wealth devoted to fight cheaters.

With these definitions, the dynamical system that describes completely the system behavior is:

$$
\begin{aligned}
\dot{Y} & =\frac{\tau_{0}}{1+\tau_{1} s \phi W}(N-Y)-\left(\alpha_{0}+\alpha_{1}(1-s) \phi W\right) Y \\
\dot{W} & =a(N-Y)-\theta W-\phi W
\end{aligned}
$$

After the normalization
$y=\frac{Y}{N} \quad w=\frac{W}{a N}$

and the re-definitions

$\tau_{1}=\tau_{1} a N \quad \alpha_{1}=\alpha_{1} N a$

the dynamical system that describes completely the system behavior is

$$
\begin{aligned}
\dot{y} & =\frac{\tau_{0}}{1+\tau_{1} s \phi w}(1-y)-\left(\alpha_{0}+\alpha_{1}(1-s) \phi w\right) y \\
\dot{w} & =(1-y)-(\theta+\phi) w
\end{aligned}
$$

The question we are facing is the following: given a social state, that is characterized by a particular setup of parameters, which is the optimal social expenditure that assures the greatest total wealth with the lowest cheaters' population?

This question can be solved by studying the steady state of the dynamical system as a function of $\phi$ and $s$. In particular, the steady cheaters' number is implicitly given by the isocline equation

$$
\tau_{0}(1-y)-\left(1+\tau_{1} s \phi w\right)\left(\alpha_{0}+\alpha_{1}(1-s) \phi w\right) y=0
$$

and steady total wealth is

$\bar{w}=\frac{1-y}{\theta+\phi}$.

Let's take a look at the following remarks:

- $\phi$ is the parameter that describes how much of the common wealth the state decides to invest.

- $s$ is the parameter that is related to how the society decides to invest its money to defeat criminality. In fact, when $s=0$ we consider only police repression while when $s=1$ society's efforts are totally directed to social promotion and education.

Figures 1 and 2 depict the tridimensional plots of both the stationary cheater population and the corresponding total wealth as a function of $\phi$ (how much) and $s$ (how). In our case, the setup of parameters is

$\alpha_{0}=0.01 \quad \alpha_{1}=1 \quad \tau_{0}=0.01 \quad \tau_{1}=100 \quad \theta=1$

As it can be seen, the cheater population decreases monotonically as $\phi$ increases for all $s \in[0 ; 1]$, the total wealth presents a peak for a given value of $\phi$ that is a function of $s$.

Consequently, the simultaneous optimization of the cheater population and the total wealth is not possible. To overcome this drawback, we can define auxiliary objective functions and we can look for the couple $(\phi ; s)$ that optimizes the strategy to fight cheaters. For our current 


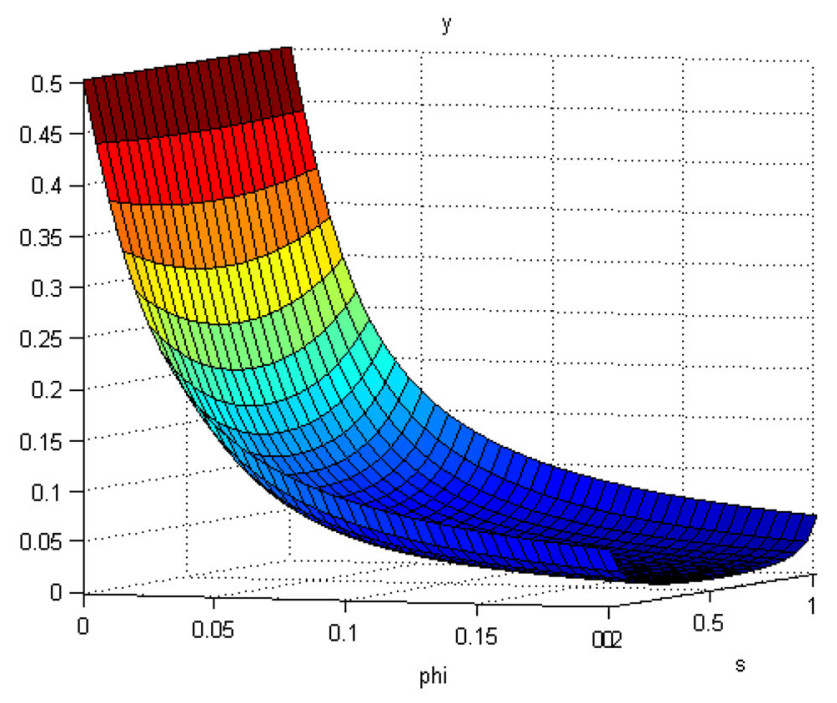

Fig. $13 \mathrm{D}$ graph of $y$ as a function of $\phi$ and $s$

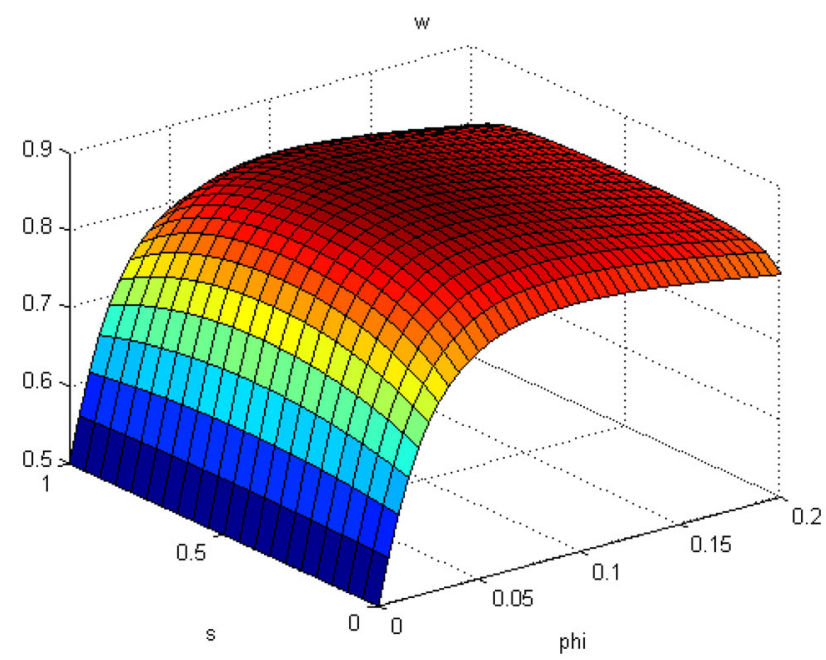

Fig. 2 3D graph of $w$ as a function of $\phi$ and $s$

purpose, a simple, but still useful choice is to study the function:

$$
L=\log \left(\frac{1}{\sqrt{(w-1)^{2}+y^{2}}}\right)
$$

Figure 3 shows the 3D graph of $L$ as a function of $\phi$ and $s$.

\section{D Cellular Automata}

We generalize the previous model to a spatial system. The spatial and temporal evolution of collective behavior in a population can be simulated with a cellular automaton model (see [9-11]).

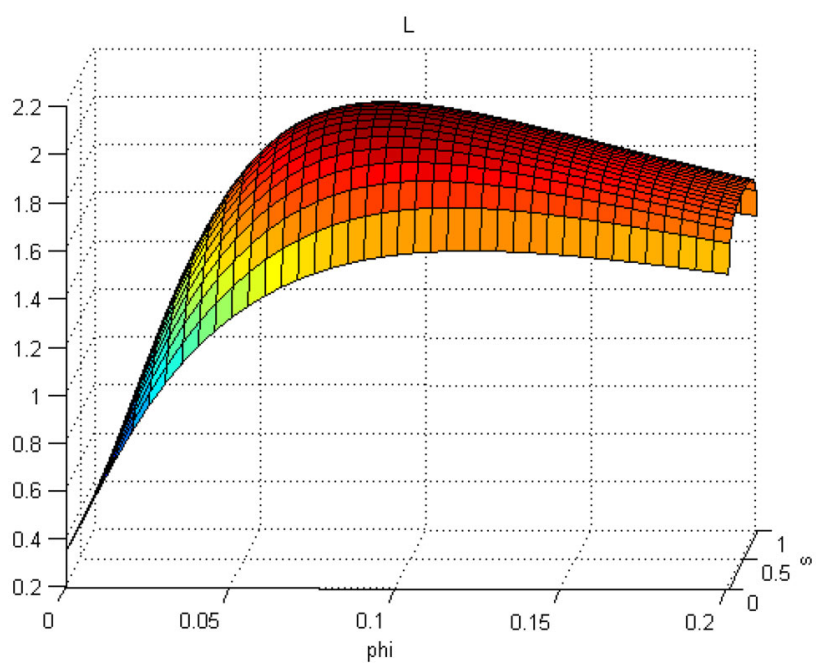

Fig. 3 3D graph of $L$ as a function of $\phi$ and $s$

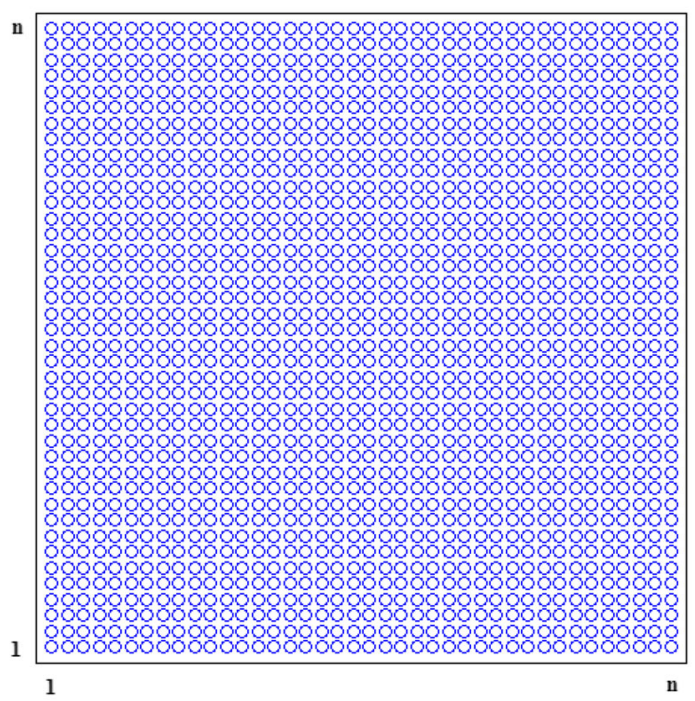

Fig. 4 The grid is a scheme of the population distributed in a square plane

Let us suppose that the population is spread in a square grid $n \times n$ as we schematized in the Fig. 4. The total number $N$ of individuals in the population is

$N=n \times n$.

Each member of the society can be a taxpayer or a cheater, that means, as we have just defined, a person who does not contribute to the common wealth but benefits from it. In the grid, if an individual is a taxpayer the point is "white" while if he is a cheater the point is "blue".

Each member of a population is influenced by the other people whom each one interacts with. Our behavior is influenced by the opinions and the actions of our neighbors and friends. On the other hand, our decisions depend on the 
global factors that are related to the whole society. We are referring to the social forces, defined as external factors which have bias opinions, that include political advertisements, news reports, laws and sanctions. Because of this, we have to take in account the effects of the so-called "micro-sociology" and "macro-sociology", to completely describe the evolution of the society. Let us first consider the "macro-sociology" influences.

The "macro-sociology" problem is related to social forces that influence the whole world. It is a sort of a global field. In fact, in its definition, we can introduce the possible actions of a state to control this form of criminality. Essentially, the possibility of a nation to fight cheaters can be divided into two strategies:

A To invest money from the common wealth to improve education and social awareness.

B To make stronger police repression (arrests). Naturally, to beef up police forces has a cost.

As we can see from the dynamic system from the previous section, the strategy $\mathrm{A}$ is related to the parameter $\tau$ to prevent through social promotion the flux from taxpayers to cheaters while strategy $\mathrm{B}$ is linked to the factor $\alpha$, forcing the flux to taxpayers trough police repression. Meacci et al. [12] introduced a probability of choosing an opinion that incorporates the global factors of the dynamic system. ${ }^{1}$ We referred to this approach to link our macrosociology parameters with the probabilities of changing behavior.

We have to define two probabilities that take into account macro-sociology parameters:

1. $P_{M}^{X \rightarrow Y}$ The probability that a taxpayer becomes a cheater;

2. $P_{M}^{Y \rightarrow X}$ The probability that a cheater becomes a taxpayer;

In the case of a taxpayer, the probability to become a cheater is defined:

$P_{M}^{X \rightarrow Y}=\tau \quad(\tau \leq 1)$

where $\tau$ is the same parameter of Eq. (4), according to the strategy A. Naturally, it is clear that the probability $P_{t}$ that a taxpayer remains a taxpayer is

$P_{M}^{X \rightarrow X}=1-P_{M}^{X \rightarrow Y}$.

In the other case, if we considered a cheater, we can define the probability to become a taxpayer as

\footnotetext{
${ }^{1}$ Differently from the present work, which begins its study from a particular ODE model and generalizes this one to a CA model introducing spatial and local effects, the paper cited, starts by defining an original CA model, compares it with the corresponding ODE model with effects of contagion and shows the advantages of the cooperation to fight tax evasion.
}

$P_{M}^{Y \rightarrow X}=\alpha \quad(\alpha \leq 1)$

where $\alpha$ is the same parameter of Eq. (5). In this case, the social force which pushes a cheater towards a honest behavior is related to the B strategy of police repression. The probability $P_{M}^{Y \rightarrow Y}$ that a cheater remains a cheater is

$P_{M}^{Y \rightarrow Y}=1-P_{M}^{Y \rightarrow X}$.

The results under the assumptions are the same as of the ODE problem. For instance, Fig. 5 shows the goal function (13) calculated with the CA model.

Let us focus on the "micro-sociology" problem. In a case of a certain probability $P$, with the notation $P(i, j)$, we indicate the probability related to the individual that lives in the position of row $i$ and column $j$ in the grid. The local probability to change opinion (or behavior) is proportional to the number of neighbors that already have different opinions (see [7]). We consider that each individual $(i, j)$ is influenced on his own and by the other 8 people close to him, as we see in Fig. 6.

These effects are called the effects of "micro-sociology" which lead to the definition of the local probability. For instance, let us suppose to define the local probability $P_{l}^{* \rightarrow Y}$ to become a cheater

$P_{l}^{* \rightarrow Y}(i, j)=\frac{n_{l}^{c}(i, j)}{N_{l}}$

where

- $n_{l}^{c}(i, j)$ is the number of people (including himself) which are the neighbors of individual $(i, j)$ who are already cheaters.

- $N_{l}$ is the total number of neighbors plus himself. It follows that $N_{l}=9$. $^{2}$

Obviously, the local probability $P_{l}^{* \rightarrow X}$ to become a taxpayer is

$P_{l}^{* \rightarrow X}(i, j)=1-P_{l}^{* \rightarrow Y}(i, j)$.

For instance, in the case of Fig. 7 the local probabilities are $P_{l}^{* \rightarrow Y}(i, j)=4 / 9$ and $P_{l}^{* \rightarrow X}(i, j)=5 / 9$.

Let us therefore define the probability of changing the current state as the convex combination of these two contributions. In particular, the total probability ${ }^{3}$ that a taxpayer became a cheater is

$P_{\mathrm{TOT}}^{X \rightarrow Y}=\gamma l P_{l}^{* \rightarrow Y}+P_{M}^{X \rightarrow Y}$

\footnotetext{
${ }^{2}$ We remark that people on the boundaries feel the influence of the people of the other side of the grid. Under this assumption the world is a sort of toros.

${ }^{3}$ We remark that the parameters will be chosen in such a way that it is a well-defined probability.
} 


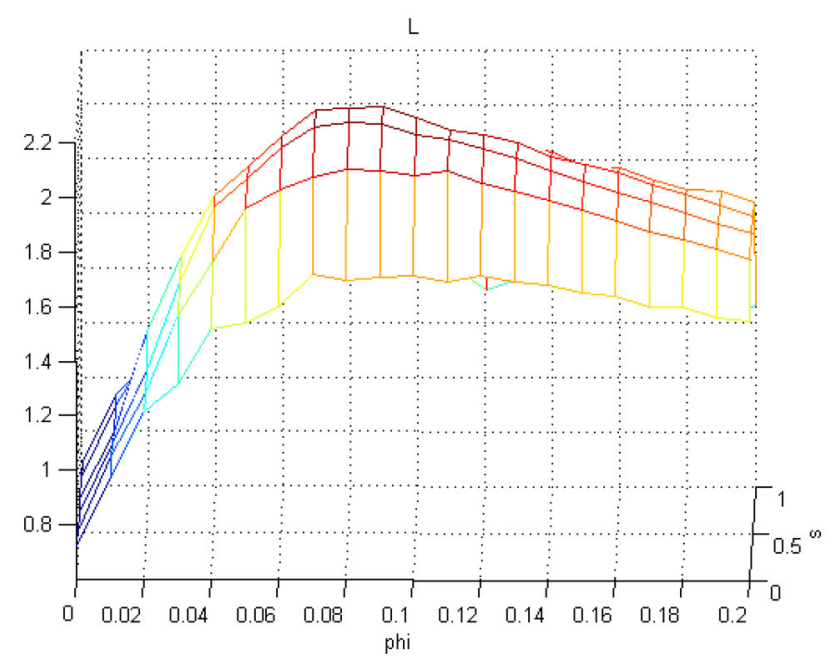

Fig. 53 graph of $L$ as a function of $\phi$ and $s$ calculated with CA model

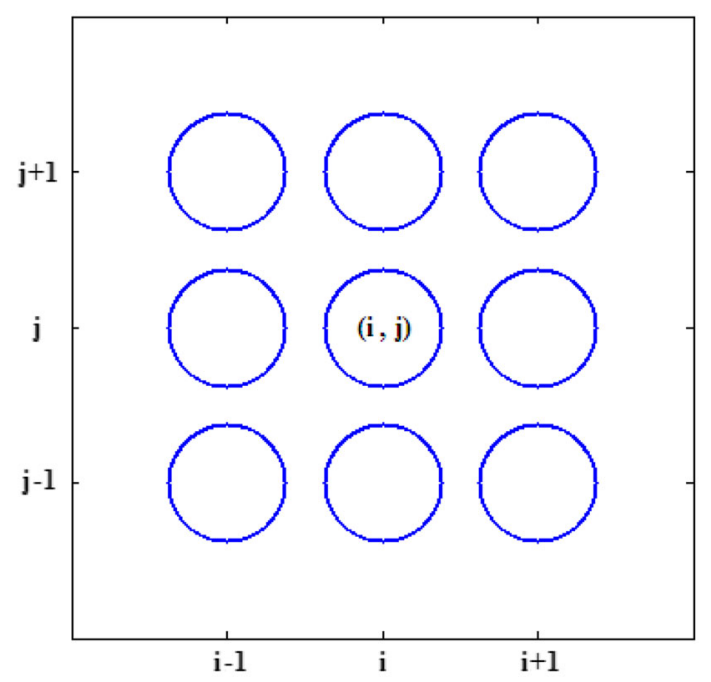

Fig. 6 An individual in position $(i, j)$ is influenced by himself and his eight neighbors

and the total probability that a cheater becomes a taxpayer is

$$
P_{\mathrm{TOT}}^{Y \rightarrow X}=\gamma k P_{l}^{* \rightarrow X}+P_{M}^{Y \rightarrow X}
$$

where $\gamma \in[0,1]$ is a parameter that allows to weigh the importance of local contributions. Essentially, tuning $\gamma$ we are exploring different types of societies according with the effectiveness of "micro-sociology" interactions. If nothing else is specified it is assumed that $\gamma=1$. Besides, $l \in[0,1]$ and $k \in[0,1]$ are 2 factors that allow to determine whether increasing the influence of contagion positive or negative. Obviously, the probability of remaining in the same state is given by $1-P_{\text {TOT }}$ in both cases.

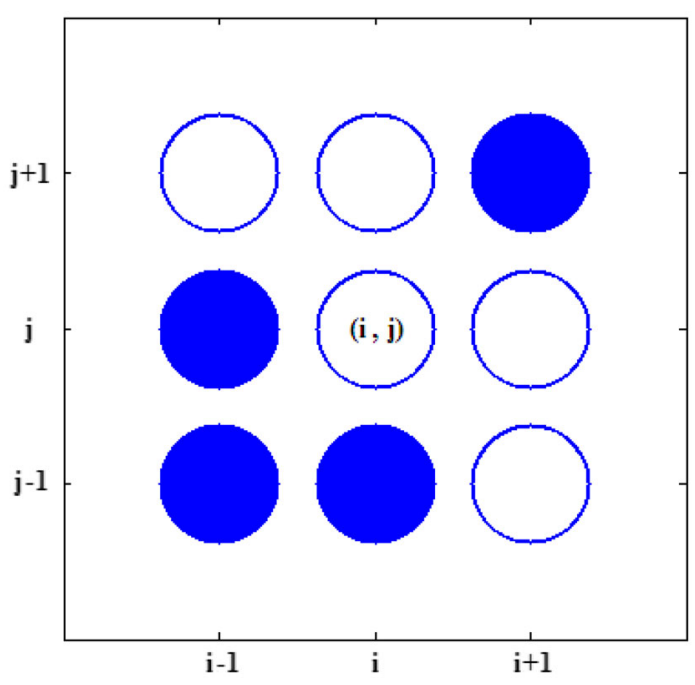

Fig. 7 The local probabilities are $P_{l}^{* \rightarrow Y}(i, j)=4 / 9$ and $P_{l}^{* \rightarrow X}(i, j)=5 / 9$
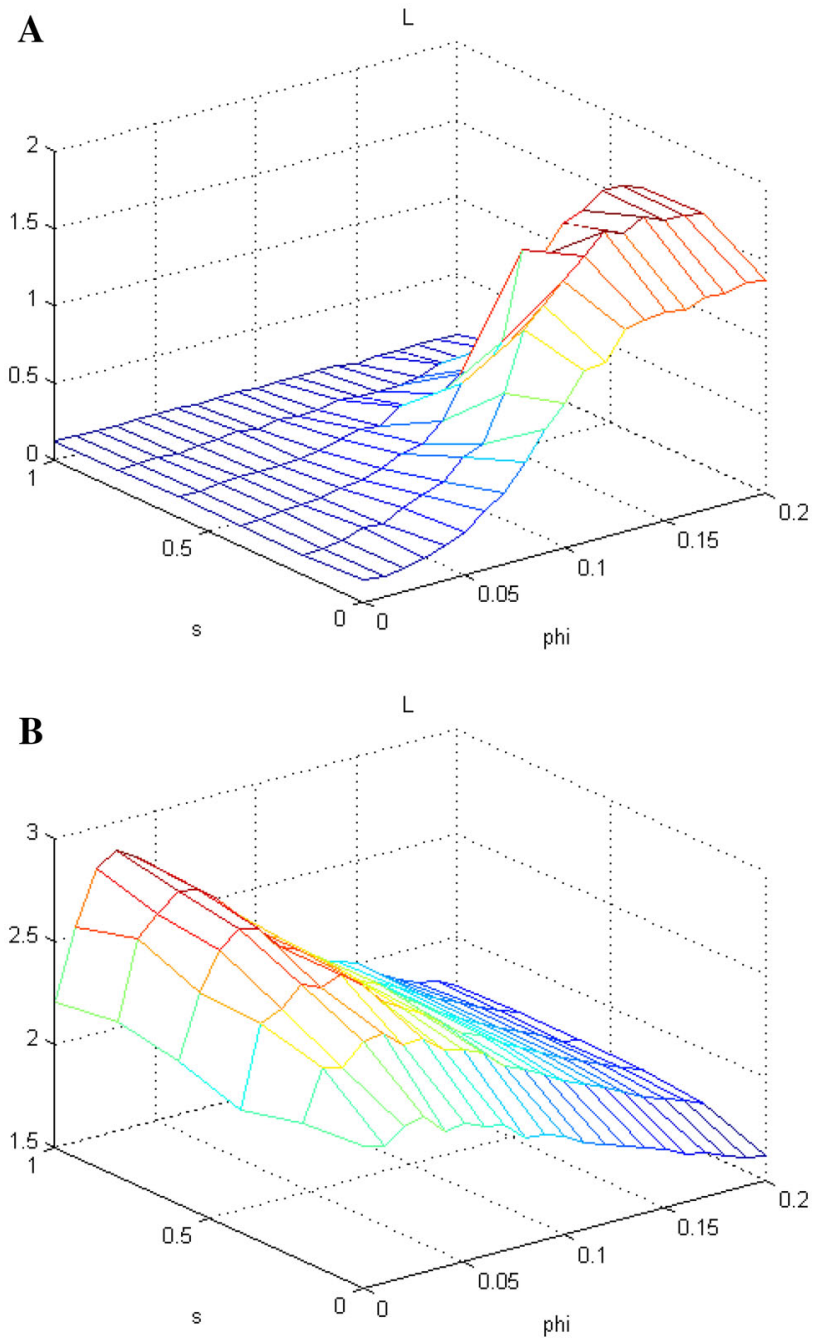

Fig. $8 L$ goal function in case a with $l=0.40$ and $k=0.30$ and in case b with $l=0.30$ and $k=0.40$ 


\section{Results and discussion}

In this section, we show the results of the CA model. These results show that the local effects have an impact on the forms of the solutions, and then the policies of optimal choice. In particular, the system is very sensitive if we decide to promote the positive or negative influence. We show in Fig. 8 the graphs of $L$ in both cases, respectively, with $l>k$ (negative case) and $l<k$ (positive case).

The CA model is particularly useful for studying the spatial effects of the system. For example, Fig. 9 shows full screenshot of the system evolution. Cheaters are identified in blue. It is evident that we can see a cluster of cheaters in the area where we have defined a lighter contrast policy $(\phi$ lower than in other regions). You can also appreciate the contagion effect in the border area of low contrast.

The effect on the system due to the influence (positive or negative) is clearly evident from Fig. 10 that shows the number of cheaters for position in a world where on the left is applied a policy of strong contrast and on the right of low contrast. The effect of infection is evident from the blunt shape of the curve, especially in the vicinity of the areas of policy change.

In fact, even if we apply different parameters in different areas of the system, by reason of the phenomenon of contagion, the effects are propagated throughout the system. Let us consider, for instance, the test shown in Fig. 11. The graph shows the density of cheaters in the world 2 where it is accompanied by the world 1 . Notice how the

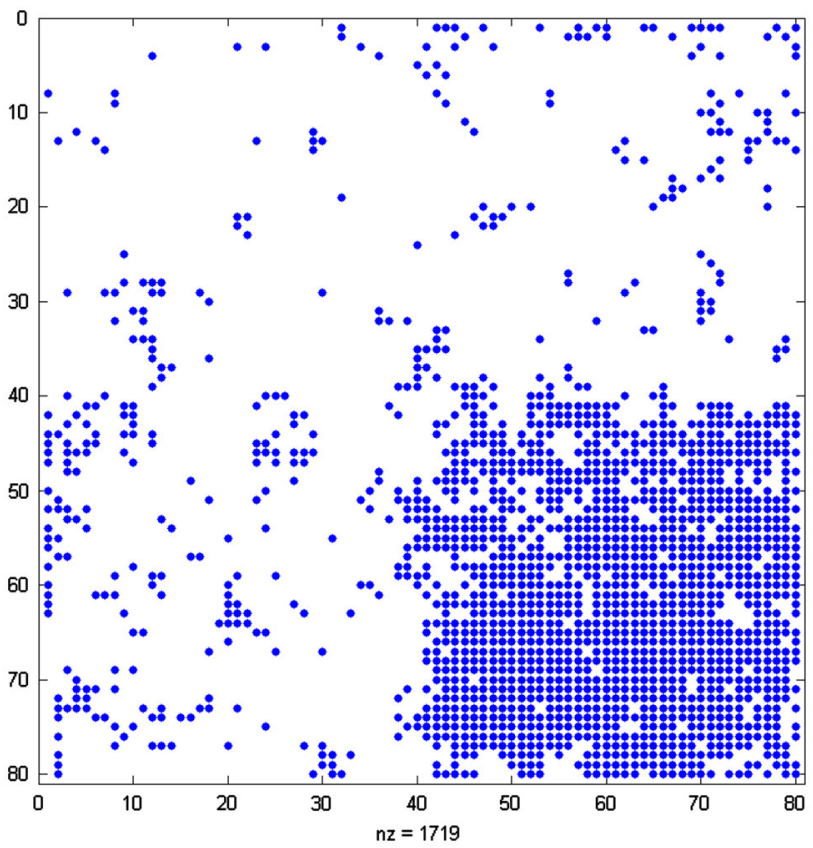

Fig. 9 A screenshot of the evolution of the system in a world where in the lower right corner we have applied a lower value of $\phi$

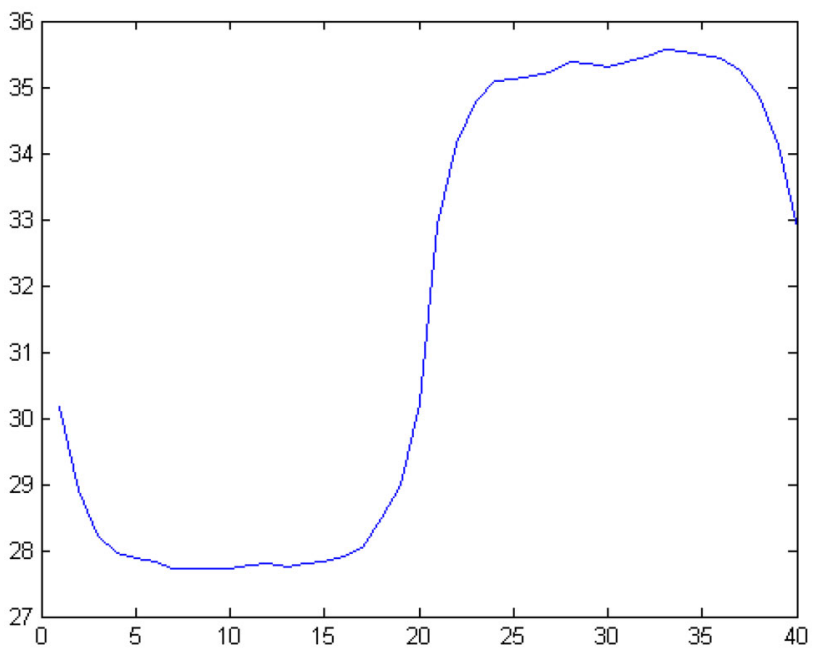

Fig. 10 Graph of the number of cheaters for position in a world in which we applied a low $\phi$ in the right area. We can appreciate the curvature of the curve due to the effect of contagion

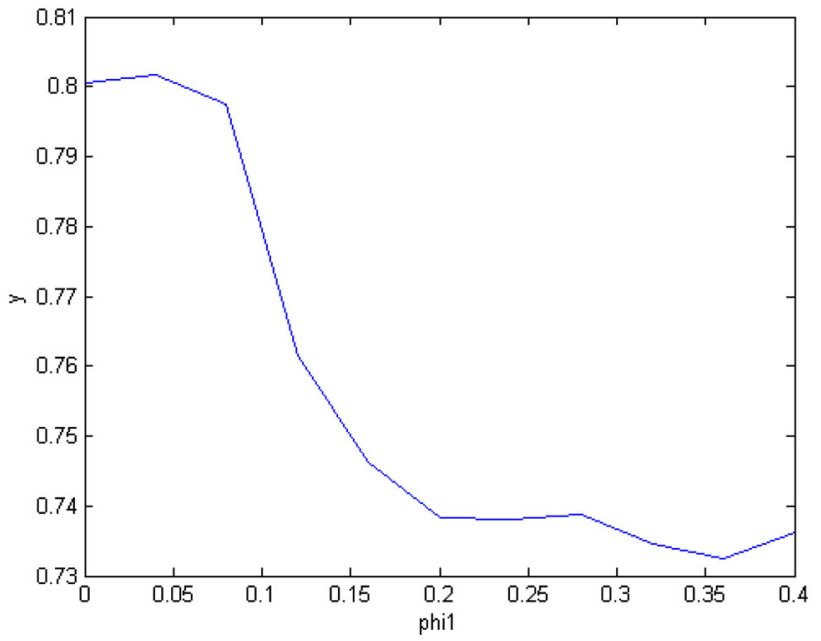

Fig. 11 Graph of the density of cheaters in the world with increasing $\phi$ of the attached world. When the $\phi$ of the attached world grows it produces a positive effect also on the world reported in the graph as a result of contagion

increase of $\phi$ in the first world, it produces a positive effect in the second world.

\section{Conclusion}

In this paper, we built a Cellular Automata model, which is consistent with the homogeneous case that corresponds to the ODE model. In particular, the CA model takes into account the "micro-sociological" effects or local effects due to the influence of contagion between individuals. These influences are particularly relevant and deeply affect 
the behavior of the system. Thanks to these influences we can study the spatial effects as shown in the "Results" section.

Acknowledgments The author wishes to thank Prof. Mario Primicerio and Prof. Juan Carlos Nuño for their contribution to bring out the ideas of the present work. The author is also grateful to Rita Adornato for checking the English of this manuscript. The paper is dedicated to Madrid, for what happened and for what will never happen.

Open Access This article is distributed under the terms of the Creative Commons Attribution 4.0 International License (http:// creativecommons.org/licenses/by/4.0/), which permits unrestricted use, distribution, and reproduction in any medium, provided you give appropriate credit to the original author(s) and the source, provide a link to the Creative Commons license, and indicate if changes were made.

\section{References}

1. Bloomquist, K.: Tax compliance as an evolutionary coordination game: an agent-based approach. Financ. Rev. 39(1), 25-49 (2011)
2. Zaklan, G., Westerhoff, F., Stauffer, D.: Analysing tax evasion dynamics via the Ising model. J. Econ. Interact. Coord. 4, 1-14 (2009)

3. Muñoz, F., Nuño, J.C., Primicerio, M.: Effects of inspections in small world social networks with different contagion rules. Physica A: Stat. Mech. Appl. 432, 76-86 (2015)

4. D'Orsogna, M.R., Perc, M.: Statistical physics of crime: a review. Phys. Life Rev. 12, 1-21 (2015)

5. Nuño, J.C., Herrero, M.A., Primicerio, M.: Fighting cheaters: how and how much to invest. Eur. J. Appl. Math. 21(4-5), 459-478 (2010)

6. Bahr, D.B.: Statistical mechanics of opinion formation and collective behavior: micro-sociology. J. Math. Sociol. 23(1), 1-27 (1973a)

7. Bahr, B.D.: Statistical mechanics of collective behavior: macrosociology. J. Math. Sociol. 23(1), 29-49 (1973b)

8. Bacaër, N.: A Short History of Mathematical Population Dynamics. Springer-Verlag, London ld (2011)

9. Wolfram, S. (ed.): Theory and Applications of Cellular Automata. World Scientific Press, Singapore (1986)

10. Kari, J.: Theory of cellular automata: a survey. Theor. Comput. Sci. 334, 3-33 (2005)

11. Chopart, B., Droz, M.: Cellular Automata Modeling of Physical Systems. Cambridge University Press, Cambridge (1998)

12. Meacci, L., Nuño, J.C., Primicerio, M.: Fighting tax evasion: a cellular automata approach. Adv. Math. Sci. Appl. 22(2), 597-610 (2012) 